\title{
Development and Validation of the Minnesota Low Vision Reading Test (MNRead) Acuity Chart for the Asante Twi Language
}

\author{
Samuel Bert Boadi-Kusi ${ }^{1}$, Michael Agyemang Kwarteng ${ }^{2}$, Emmanuel Asubonteng ${ }^{3}$
}

1. Department of Optometry and Vision Science, School of Allied Health Sciences, University of Cape Coast, Ghana. 2. Discipline of Optometry, University of KwaZulu-Natal, Durban, South Africa.

3. Department of Ghanaian Languages and linguistics, Faculty of Arts, University of Cape Coast, Ghana.

\begin{abstract}
Aim: To design and validate a MNREAD Asante Twi version reading chart, to aid in the assessment of near visual acuity of natives Ghanaians.

Methods: Cross-sectional and experimental designs were employed in phases I and II respectively of this study. The chart was developed using 20 transited pupils in class four in the Kumasi metropolis in phase I and validated in a clinical setting at the Manhyia District Hospital, Kumasi, using students in phase II.

Results: A total of 100 participants (mean age; $22.19 \pm 1.61$ years) were involved in the second phase of this study. A Pearson product-moment correlation coefficient was computed to assess the relationship between MNREAD Asante Twi charts $\log$ MAR scores in both eyes. The MNREAD-Asante Twi acuity charts had very strong correlations $(r=0.94, p<0.001)$ for MNREAD set 1A (black on white background) scores and MNREAD set 2A (black on white background) for acuity scores. Correlation between MNREAD set 1B (white on black background) scores and MNREAD set $2 \mathrm{~B}$ (white on black background) scores was $\mathrm{r}=0.95, \mathrm{p}<0.001$.

Conclusion: MNREAD Asante Twi reading chart will enhance the measurement of near visual function in native Ghanaians.

Keywords: Asante Twi, MNREAD, Visual Acuity, Near chart, Ghana.

DOI: https://dx.doi.org/10.4314/ahs.v19i4.15

Cite as: Boadi-Kusi SB, Kwarteng MA, Asubonteng E. Development and Validation of the Minnesota Low Vision Reading Test (MNRead) Acuity Chart for the Asante Twi Language. Afri Health Sci. 2019;19(4):2945-2953. https://dx.doi.org/10.4314/abs. v19i4.15
\end{abstract}

\section{Introduction}

In the assessment of near visual acuity, measurements based on a single optotype are not adequate indicators of reading performance because the reading function is a highly complex ability apart from good acuity. This function is a combination of sensual (such as visual acuity and contrast sensitivity), motor (such as eye movements), and cognitive abilities. ${ }^{1}$ Print size is the most important factor that affects reading speed in individuals with normal and low vision., ${ }^{2,3}$ Therefore, continuous-text reading acuity charts with proven validity and reliability are required for the assessment of reading performance, rather than single optotype measurements. ${ }^{4}$

\section{Corresponding author:}

Samuel Bert Boadi-Kusi,

Department of Optometry and Vision Science, School of Allied Health Sciences, University of Cape Coast, Ghana.

Email: sboadi-kusi@ucc.edu.gh
The MNREAD Acuity charts are continuous-text reading-acuity charts which have been developed over the years for assessing the reading acuity and reading speed of normal and low-vision patients. ${ }^{2}$ The first of its kind was developed by Legge and his colleagues ${ }^{2}$ at the Minnesota Low-Vision Centre using short sentences. ${ }^{2,3}$ They are designed to measure reading acuity, critical print size, and maximum reading speed. They are reliably used in clinical applications and in scientific research. The concepts of standard-length words and 60-character sentence have been adapted in the scoring of the MNREAD charts to minimize differences in scoring. ${ }^{1-3,5-7}$ Charts include 19 logarithmic sentences in the logMAR range of -0.5 to 1.3, with 0.1 logarithmic intervals. These concepts and the design make the MNREAD charts preferred for the visual assessment of normal and low vision patients. MNREAD charts also show the equivalent Snellen and $M$ values, in addition to logMAR. ${ }^{1-3,5-7}$

Ghana has an illiterate population of about $38.3 \%$ of its total population of $28,308,301 .{ }^{8}$ The assessment of near

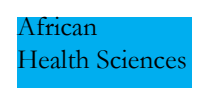

(C) 2019 Boadi-Kusi et al. Licensee African Health Sciences. This is an Open Access article distributed under the terms of the Creative commons Attribution License (https://creativecommons.org/licenses/BY/4.0), which permits unrestricted use, distribution, and reproduction in any medium, provided the original work is properly cited.

African Health Sciences Vol 19 Issue 4, December, 2019 
visual acuity in this population is based on the "Tumbling E" which only measures good acuity and in few instances on the single letter reduced Snellen Acuity charts which are English-based. Although most of these near visual acuity assessments focus on presbyopia correction, this correction is mainly concerned with the goal of regaining an uncorrected reading performance. ${ }^{9}$

Asante Twi is one of the dialects of the Akan language which is the most widely spread language spoken by about $47.5 \%$ of the total population of Ghana. ${ }^{8}$ Most of the illiterate indigenous people are able to read the Akan text although they may not be able to read and write in the English language. ${ }^{8}$ With the emergence of MNREAD English reading charts, effort has been made to produce a similar chart in different languages such as Portuguese ${ }^{5}$, Greek $^{6}$, Turkish ${ }^{7}$ among others with the view of measuring all the components of near visual acuity. This study was therefore aimed at designing and validating an $\mathrm{MN}$ READ near acuity chart using the most popular Ghanaian language (Akan text) that would be useful in terms of international comparability in clinical applications and scientific research, in which near vision and reading performance could be appraised.

\section{Methods}

Cross-sectional and experimental study designs were employed in phases I and II of this study respectively. The two phases were i. developing the near reading chart, which was undertaken among transited pupils in class four in the Kumasi metropolis of the Ashanti Region of Ghana. ii. validating the tool in a clinical setting at the Manhyia District Hospital, Kumasi, of the Ashanti region of Ghana using student-patients. Questionnaires were used to collect demographic data of participants in both phases.

\section{Sampling and recruitment of participants}

A purposive sampling technique was used to select 20 class four pupils due to the fact that they had recently transited to the upper primary class at the time of the study. The selection of 20 pupils was to ascertain that the Twi sentences were legible and readable by most participants. Secondly, a purposive sampling was used to select 100 student-patients from the Manhyia District Hospital, who were literate in both English and Asante Twi to validate the near reading chart in a clinical setting.

\section{Inclusion and Exclusion Criteria}

The inclusion criteria were as follows; i. Individuals who were fluent in both English and Asante Twi.

ii. Subjects with habitual distance visual acuity of $20 / 20$ or better and near visual acuity of at least N.5.

iii. The absence of active ocular pathology, including strabismus.

iv. The absence of any history of a reading problem or learning difficulties.

Subjects studying Ghanaian languages and linguistics with Asante Twi option were excluded as this would have influenced the outcome.

\section{Development of the chart}

One hundred sentences were constructed from Ghanaian Basic School (Class 2 and Class 3) Twi textbooks which were recommended by the Ghana Educational Service (GES). The one hundred Asante Twi sentences were constructed by the researchers and a team of language experts from the Department of Ghanaian Languages and linguistics of the University of Cape Coast. Out of the 100 sentences, 60 met the criteria using the recommended software by the Minnesota Low Vision Centre to assess the number of characters, length, and width of the alphabets. ${ }^{1,25-7}$ The near $\log$ MAR chart required a set of 19 sentences for one chart and therefore 60 sentences were enough to facilitate the selection of easy sentences for the development of two sets of the chart. The selected sentences had 60 characters including spaces and periods after the sentences. The selection of the number of characters per sentence was convenient for scoring since a "standard-length word" had 6 characters with the assumption made to the effect that the 60-character sentence consisted of 10 standard-length words. These made the measurement of near visual acuity in logMAR easier by making a "standard-length word" score a 0.01 point.

\section{Testing procedure using the basic school pupils}

1. Participants were requested to read the sentences aloud one after the other, starting from the first sentence to the last using both eyes.

2. A time interval of 2 minutes was given to participants to rest after reading every ten sentences.

3. The time used to read each sentence was taken during the reading of the sentences.

4. Participants were instructed to continue reading regardless of any error that was made prior to the start of the exercise.

5. The most readable and legible 38 sentences out African Health Sciences Vol 19 Issue 4, December, 2019 
of the 60 sentences were selected for the construction of the charts. A chart comprises 19 sentences and hence two sets of chart required 38 sentences. The rationale behind this method is that the children spent much time on words that were difficult to pronounce resulting in longer reading time.

\section{Measuring reading time}

The reading time of the sentences was determined in seconds with a stopwatch and this was used to estimate the reading performance of participants as well as the legibility and readability of sentences. The first 38 sentences that required least time to read were selected.

\section{Measuring reading error}

Sentences with difficult words had much longer reading time. Such sentences were not included in the construction of the charts.

\section{Validation of the chart}

The 38 sentences obtained from the first phase of the study were used in the development of the logMAR near reading charts. The logMAR near reading chart in Asante Twi contained 19 sentences with different sizes. The print size steps between successive sentences followed a logarithmic progression. The difference in the print size of the sentences ranged from 1.3 to -0.5logMAR (Snellen 20/400 to 20/6). Snellen, N, and M notations were provided along the logMAR chart design for easy conversion. A Times Asante Twi font was used and the sentences were printed on an A2 hard sheet. A high contrast $(85 \%)$ and a standard luminance of $80-90 \mathrm{~cd} / \mathrm{m}^{2}$ were employed. The 38 sentences were randomly selected for the development of the two charts.

Demographic data of participants were obtained through a self-administered questionnaire. Following this, clinical assessment was conducted to ensure that participants met the inclusion criteria for the validation phase. Participants were then taken through the procedure outlined below.

\section{Testing procedure in the clinic}

1. Participants were requested to read the sentences of the randomized logMAR charts; MNREAD Asante Twi set $1 \mathrm{~A}$ (black on white background), 2A (black on white background) and, 1B (white on black background) or $2 \mathrm{~B}$ (white on black background) aloud one after the other, starting from logMAR 1.00 till the participant could not read anymore. $\operatorname{LogMAR} 1.00$ was the start point rather than logMAR 1.30 since the participants had normal vision and $\operatorname{logMAR} 1.30$ is developed for low vision patients. This was done for both eyes only.

2. The time used to read each sentence was taken during the reading.

3. Participants were encouraged to continue reading regardless of any error that was made.

The reading acuity was determined by estimating the smallest print size that a participant could read the entire sentence without making significant errors. Each sentence had 60 characters which correspond to 10 standard length words, and assuming a standard word length is of 6 characters. ${ }^{5-7}$ The reading acuity was made against each sentence to make a recording of reading acuity easy. The acuity was determined as follows;

Acuity $=1.1-($ sentences $\times 0.1)+($ errors $\times 0.01) .{ }^{1,2}$

Reading speed was measured in words per minute. Since each sentence is assumed to have 10 standard length words, Reading speed $=600 /$ (time in seconds).$^{1,2}$

The reading speed was taken alongside the measurement of the reading acuity. A stopwatch was used to measure the reading speed.

\section{Data analysis}

Data were analyzed using the SPSS version 22 (Chicago, IL, USA). A test of central tendency was performed on the data (i.e. descriptive measures such as mean, standard deviation and range). A Pearson product-moment correlation and paired T-test were utilized in the analysis. The Correlation and paired T-test were performed on the data to test for statistical significance. This was done to determine if there were differences in the logMAR scores of MNREAD Asante Twi charts. Repeatability of test results was done using its own reversed-contrast charts and was analysed using paired T-test. ${ }^{11-14}$

\section{Ethical consideration}

The study was approved by the Institutional Review Board of the University of Cape Coast, Cape Coast with ethical clearance ID No: (UCCIRB/CHAS/2015/092) and adhered to the tenets of the Declaration of Helsinki.

\section{Results}

\section{Demographic characteristics of participants}

Twenty participants were involved in phase I of the study. In this phase, sentences were constructed for the design of the MNREAD Asante Twi charts. There were more females $(55 \%)$ in this cohort and the mean age of the sample population was $9.95 \pm 1.05$ years. A total of 100 subjects were involved in phase II of the study. The mean 
age of participants in this phase was $22.19 \pm 1.61$ years. There were more females (56\%) in the study sample population.

\section{Validation of Asante Twi chart}

A Pearson product-moment correlation coefficient was computed to assess the relationship between MNREAD set 1A (black on white background), 1B (white on black background), 2A (black on white background) and 2B (white on black background) logMAR scores in both eyes. There were strong positive correlation coefficients $(r=0.94 ; r=0.95)$ between the MNREAD set $1 \mathrm{~A}$ and $2 \mathrm{~A}$ sets; and $1 \mathrm{~B}$ and $2 \mathrm{~B} \log \mathrm{MAR}$ scores respectively as shown in Table 1. Also, there were strong correlations, $(\mathrm{r}=0.88 ; \mathrm{r}=0.91)$ for maximum reading speed between Set $1 \mathrm{~A}$ and Set $2 \mathrm{~A}$; and $1 \mathrm{~B}$ and $2 \mathrm{~B}$ respectively (Table 2 ), and $r=1.0$ for critical print size at $0.2 \log$ MAR for all the sets (Figure 1-4).

Table 1: Correlation test and coefficient among charts

\begin{tabular}{|c|c|c|c|c|}
\hline LogMAR Acuity & $\mathrm{N}=100$ & Correlation & Sig. & $\overline{\mathrm{R}^{2}}$ \\
\hline 1 & $\begin{array}{l}\text { OU MNREAD SET 1A SCORE \& } \\
\text { OU MNREAD SET } 2 \text { A SCORE }\end{array}$ & .942 & .000 & 0.89 \\
\hline 2 & $\begin{array}{l}\text { OU MNREAD SET 1B SCORE \& } \\
\text { OU MNREAD SET 2B SCORE }\end{array}$ & .949 & .000 & 0.90 \\
\hline 3 & $\begin{array}{l}\text { OU MNREAD SET 1A SCORE \& } \\
\text { OU MNREAD SET 1B SCORE }\end{array}$ & .624 & .000 & 0.39 \\
\hline 4 & $\begin{array}{l}\text { OU MNREAD SET 2A SCORE \& } \\
\text { OU MNREAD SET } 2 \mathrm{~B} \text { SCORE }\end{array}$ & .618 & .000 & 0.38 \\
\hline
\end{tabular}




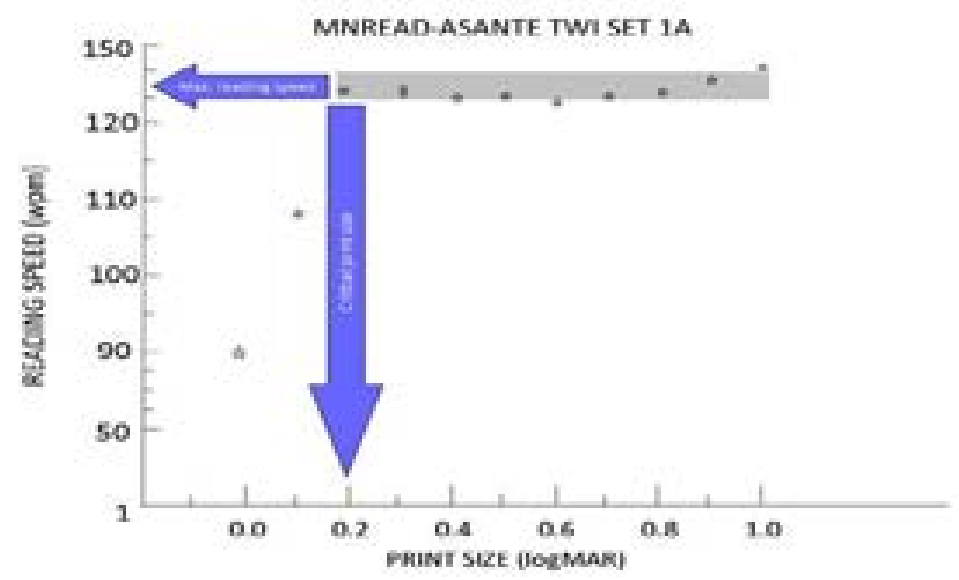

Figure 1: Reading Speed versus Print Size of MNREAD Asante Twi charts 1A.

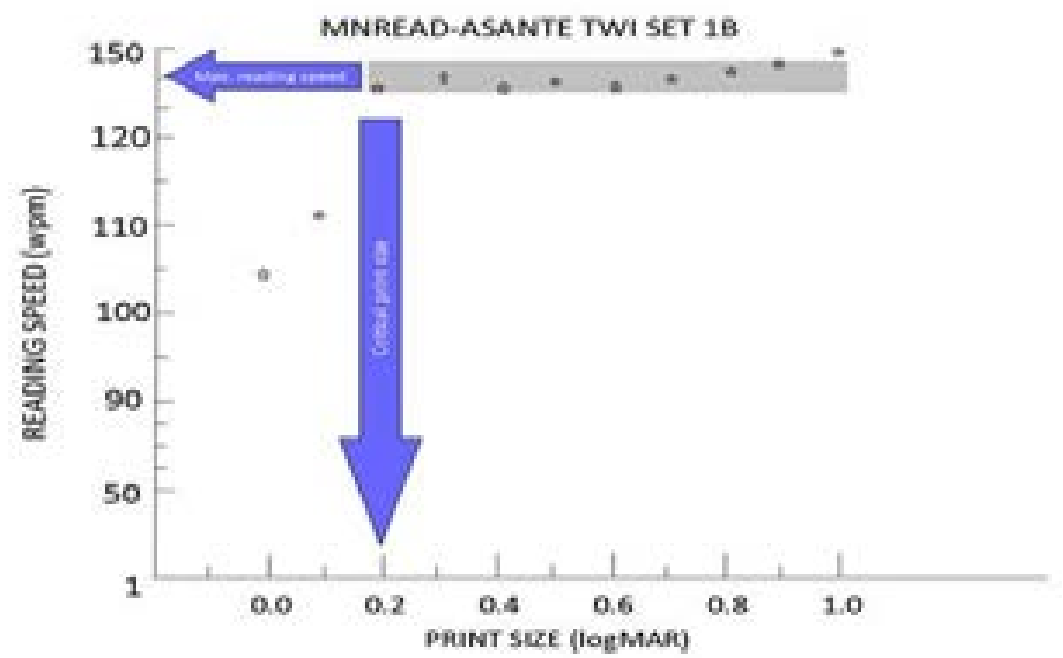

Figure 2: Reading Speed versus Print Size of MNREAD Asante Twi charts 1B. 


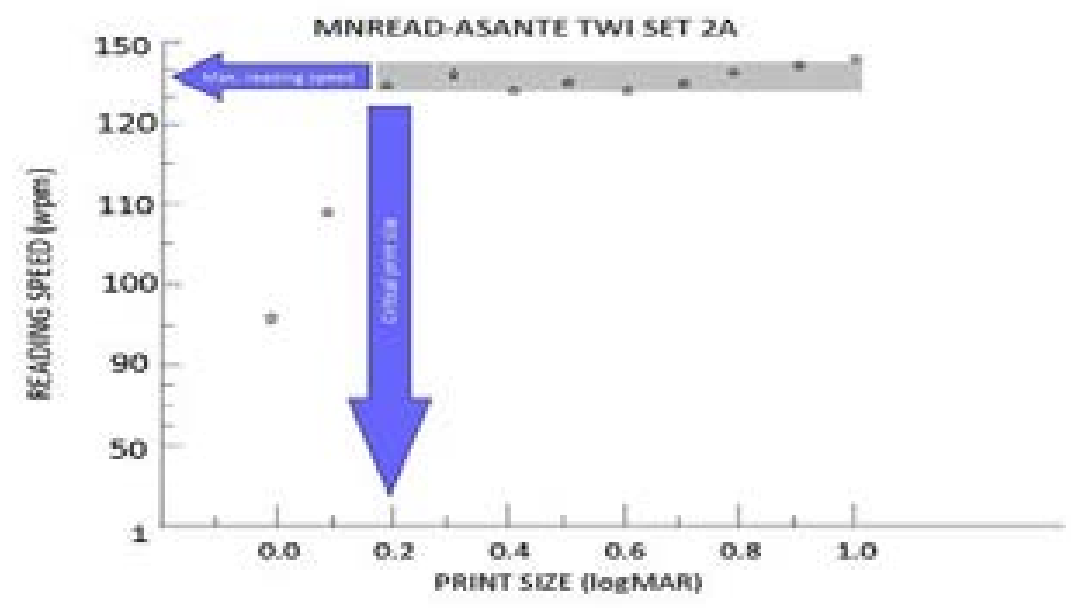

Figure 3: Reading Speed versus Print Size of MNREAD Asante Twi charts 2A.

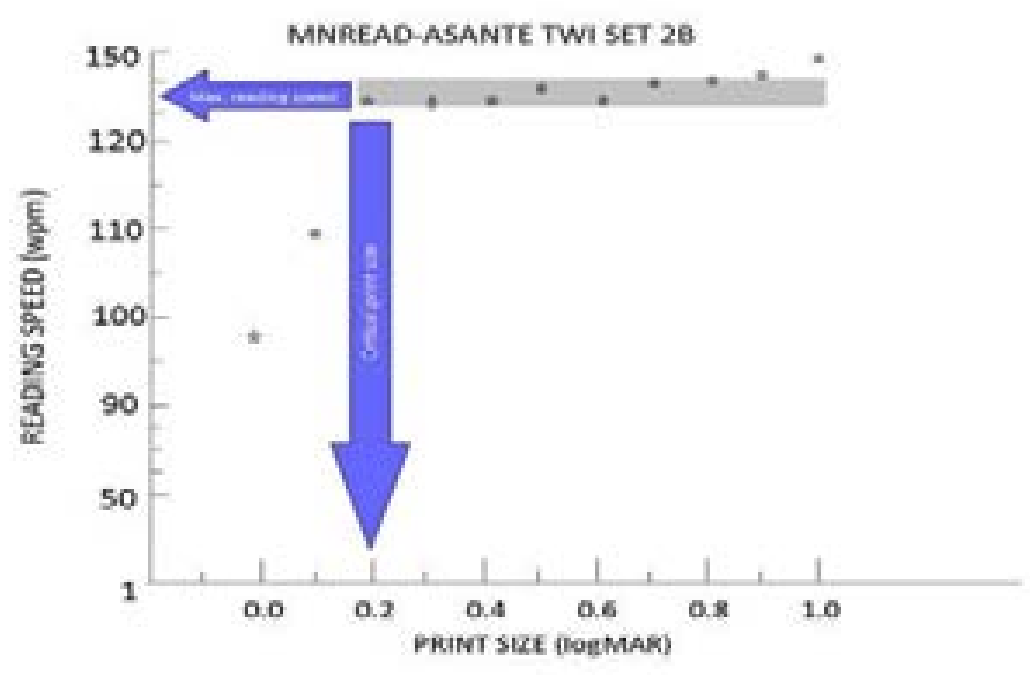

Figure 4: Reading Speed versus Print Size of MNREAD Asante Twi charts 2B.

There were no statistically significant differences $(\mathrm{P}>$ 0.05) between the mean MNREAD set $1 \mathrm{~A}$ (black on white background) and mean MNREAD set 2A (black on white background) scores and mean MNREAD set $1 \mathrm{~B}$ (white on black background) and mean MNREAD set 2B (white on black background) scores as shown in Table 3. There were statistically significant differences $(p<0.05)$ between the mean MNREAD set $1 \mathrm{~A}$ (black on white background) and mean MNREAD set $1 \mathrm{~B}$ (white on black background) scores and mean MNREAD set 2A (black on white background) and mean MNREAD set $2 \mathrm{~B}$ (white on black background) scores as shown in
Table 3.

Finally, the MNREAD-Asante Twi acuity reverse contrast charts (repeatability) had moderate correlations, $r=$ 0.62 for MNREAD set $1 \mathrm{~A}$ scores and MNREAD set $1 \mathrm{~B}$ scores, $r=0.89$ for maximum reading speed (Tables $1 \&$ 2 ), $r=1.0$ for critical print size at $0.2 \log$ MAR (figures $1 \& 2$ ) and the coefficient of determination, $r^{2}=0.39$. Correlation between MNREAD set $2 \mathrm{~A}$ scores and MNREAD set $2 B$ scores was $r=0.62, r=0.82$ for maximum reading speed (Table $1 \& 2$ ) $\mathrm{r}=1.0$ for critical print size at $0.2 \log$ MAR (Figures $3 \& 4$ ) and the coefficient of determination, $\mathrm{r}^{2}=0.38$. 
Table 2: Correlation of means of maximum reading speed

\begin{tabular}{|c|c|c|c|c|c|}
\hline & F MAXIMUM READING SPEED & $\mathrm{N}=100$ & Correlation & Sig. & MEAN \pm SD \\
\hline 1 & $\begin{array}{l}\text { OU MNREAD SET 1A \& } \\
\text { OU MNREAD SET 2A }\end{array}$ & & .876 & .002 & $\begin{array}{l}132.26 \pm 4.71 \\
137.33 \pm 4.05\end{array}$ \\
\hline 2 & $\begin{array}{l}\text { OU MNREAD SET 1B \& } \\
\text { OU MNREAD SET } 2 B\end{array}$ & & .914 & .001 & $\begin{array}{l}139.66 \pm 4.40 \\
134.59 \pm 4.71\end{array}$ \\
\hline 3 & $\begin{array}{l}\text { OU MNREAD SET 1A \& } \\
\text { OU MNREAD SET 1B }\end{array}$ & & .893 & .001 & $\begin{array}{l}132.26 \pm 4.71 \\
139.66 \pm 4.40\end{array}$ \\
\hline 4 & $\begin{array}{l}\text { OU MNREAD SET 2A \& } \\
\text { OU MNREAD SET 2B }\end{array}$ & & .821 & .007 & $\begin{array}{l}137.33 \pm 4.05 \\
134.59 \pm 4.71\end{array}$ \\
\hline
\end{tabular}

Table 3: Mean difference among charts

\begin{tabular}{llll}
\hline LogMAR Acuity & Mean & Std. Deviation & Sig. (2-tailed) \\
\hline $\begin{array}{l}\text { OU MNREAD SET 1A SCORE \& } \\
\text { OU MNREAD SET 2A SCORE }\end{array}$ & .0001 & 0.02 & .567 \\
$\begin{array}{l}\text { OU MNREAD SET 1B SCORE \& } \\
\text { OU MNREAD SET 2B SCORE }\end{array}$ & -0.001 & 0.02 & .666 \\
$\begin{array}{l}\text { OU MNREAD SET 1A SCORE \& } \\
\text { OU MNREAD SET 1B SCORE } \\
\text { OU MNREAD SET 2A SCORE \& }\end{array}$ & -0.06 & 0.05 & .000 \\
OU MNREAD SET 2B SCORE & -0.06 & 0.05 & .000 \\
\hline
\end{tabular}

\section{Discussion}

This is the first MNREAD-Asante Twi reading chart developed and validated to assess the visual function of normal and low vision patients of native Akan readers. It will be a useful predictor for many visual problems such as presbyopia in native Ghanaians.The MNREAD-Asante Twi acuity charts had very strong correlations for MNREAD set $1 \mathrm{~A}$ scores and MNREAD set $2 \mathrm{~A}$ scores, maximum reading speed, critical print size at $0.2 \log M A R$ and high coefficient of determination for acuity scores. Similarly, the correlation between MNREAD set $1 \mathrm{~B}$ scores and MNREAD set $2 \mathrm{~B}$ scores were strong, with a similarly strong correlation for maximum reading speed for critical print size at $0.2 \log \mathrm{MAR}$ and the coefficient of determination was equally high.

In the validation of a Persian reading chart, correlation coefficient and specificity were the main validity tests performed to assess the reliability of the developed Persian chart. ${ }^{10}$ The MNREAD-Asante Twi acuity charts were considered as its own references for the validation since all the principles recommended by the Minnesota Low Vision Centre were met. ${ }^{1,5-7}$ The results of the mean scores showed that clinically, there were no significant differences between the mean MNREAD set 1A (black on white background) and mean MNREAD set 2A (black on white background) scores and mean MNREAD set 
1B (white on black background) and mean MNREAD set 2B (white on black background) scores as shown in Table 3 because the difference did not exceed $0.1 \log$ MAR.

The MNREAD-Asante Twi acuity charts reliability and repeatability were considered with its own reversed-contrast charts using the mean differences between the acuity scores. There were statistically significant differences $(\mathrm{p}<0.05)$ between the mean MNREAD set 1A (black on white background) and mean MNREAD set $1 \mathrm{~B}$ (white on black background) scores and mean MNREAD set $2 \mathrm{~A}$ (black on white background) and mean MNREAD set 2B (white on black background) scores. However, the results showed that clinically, there were no significant differences between the mean MNREAD set 1A (black on white background) and mean MNREAD set $1 \mathrm{~B}$ (white on black background) scores and mean MNREAD set 2A (black on white background) and mean MNREAD set $2 \mathrm{~B}$ (white on black background) scores because the difference was approximately $0.1 \operatorname{logMAR}$ as shown in Table 3 . This is supported by a recent MNREAD study in which the coefficient of repeatability for MNREAD-Greek tests was $0.08 \log \mathrm{MAR} .{ }^{5}$ A $\log \mathrm{MAR}$ visual acuity score needs to change by more than two lines ( $0.2 \log$ MAR) to be considered as a clinically significant change. ${ }^{11-14}$ In spite of this, in a recent study, repeatability tests that have a change of $0.03 \pm 0.09 \log$ MAR in visual acuity are accepted. ${ }^{13}$ In most studies, the reported test-retest errors were within 0.04 to $0.06 \log$ MAR. ${ }^{15-19}$ Thus, a change of 1 line or 0.1 $\log$ MAR is suggested to be clinically significant in normal adults. ${ }^{15-19}$ A related study reported a coefficient of repeatability of $0.08 \log \mathrm{MAR}$ for VA, 46.96 words per minute for maximum reading speed and $0.10 \log$ MAR for critical print size. ${ }^{5}$ These results are consistent with those of the current study.

Although it was expected that the correlation and coefficient of determination for the reverse contrast would be high, they were moderate and low respectively in this study. External factors such as the spatial direction of alphabets, difficulty in printing the reverse charts at the design stage among others might have influenced the measurement of the acuity score. This finding is very common in non-English reading cards ${ }^{20}$ since the spatial direction of alphabets and different forms of them in different words need different visual processing. ${ }^{4}$

Legibility, literacy difficulties, context themes and statistical frequencies of words legibility are of great impor- tance in the development of a near reading chart. ${ }^{21}$ In this study, simple texts were considered and each sentence was about a different subject and the relative sentence legibility was determined using reading time. The designing of the chart took into consideration contrast sensitivity and illumination. High contrast (85\%) black letters were presented on two sets of white background and two sets of the black background on (A-2 print) sheets. A standard luminance of $80-90 \mathrm{~cd} / \mathrm{m} 2$ was used as proposed by Radner. ${ }^{22}$

A near reading chart that resembles real reading conditions is important to assess the quality of life in terms of reading performance. Measuring reading speed and reading acuity are considered important in the clinical diagnosis of visual function anomalies. The development of a standardized and validated reading test $\mathrm{t}^{22}$ in Asante Twi is important to allow reliable evaluation of reading performance among native Ghanaians who are fluent in Asante Twi. In this study, all the participants were Ghanaians and their native language was Asante Twi. They learned Asante Twi as a course at the basic education level. Therefore, as shown in Figures 1- 4, graphs of reading speed versus print size with the MNREAD charts revealed a high reading performance of the participants. The reading speed measurement using the Asante Twi Reading Chart in the current study was similar to the one reported by Legge et al. ${ }^{23}$ using the MNREAD Acuity Chart. Legge et al. ${ }^{23}$ classified reading performance into three groups; fluency 160 words $/ \mathrm{min})$, functional $(80 \leqslant$ words $/ \mathrm{min}<160)$ and Slow ( $<80$ words/min). While Legge et al. ${ }^{23}$ measured fluency ( $\geqslant 160$ words/min) reading performance, while the MNREAD-Asante Twi recorded functional (80 $\leqslant$ words $/$ min $<160$ ) reading performance. In this study, the maximum reading speed with the MNREAD-Asante Twi was in the functional reading performance category. This reflected the real situation of the participants.

The authors acknowledge the limited pool of Asante Twi sentences that was available for selection and the difficulty in printing the reverse chart as limitations of this study. However, these did not impact negatively on the outcome of the study results. Further scientific research is needed on the subject matter in Ghana.

\section{Conclusion}

MNREAD-Asante Twi reading chart will help to deter- 
mine the near visual function, reading speed and critical print size of the local people in Ghana, specifically those who speak Asante Twi. It will also enhance the measurement of near visual function in normal and low-vision native patients of native Akan people in Ghana.

\section{Acknowledgement}

The authors are grateful to Prof Steve Mansfield of the Department of Psychology, State University of New York College at Plattsburgh for providing us the software for the MNREAD characters, commenting on the analysis and his guidance during the development of the chart.

\section{Conflict of interest}

The authors declare that there are no conflicts of interest.

\section{Reference}

1. University of Minnesota Laboratory of Low Vision Research. MNREAD-History. Available at www.gellab. dl.umn.edu/MNREAD-history. Accessed on March 28, 2017.

2. Mansfield JS, Ahn SJ, Legge GE, Luebker A. A New Reading Chart for Normal and Low Vision. Ophthal Vis Opt / Non-inva Ass Vis Syst Tech Dig. 1993;3:232-35.

3. Legge GE, Ross JA, Luebker A, LaMay JM. Psychophysics of Reading VIII. The Minnesota Low-Vision Reading Test. Optom Vis Sci. 1998;66:843-53.

4. MacMonnies CW. Chart Construction and Letter Legibility/Readability. Ophthal Physiol Opt. 1999;19:498506.

5. Tamaki C, Kallie CS, Legge GE, Salomao SR, et al. Validation of the MNREAD-Portuguese Continuous-Text Reading-Acuity Chart. Invest Ophthalmol Vis Sci. 2004;45:43-58

6. Mataftsi A, Bourtoulamaiou A, Haidich AB, et al. Development and Validation of the Greek Version of the MNREAD Acuity Chart. Clin Exp Optom. 2013;96: 25-31. 7. İdil SA, Caliskan D, İdil NB. Development and Validation of the Turkish Version of the MNREAD Visual Acuity Charts. Turk J Med Sci. 2011;41:565-70.

8. Ghana Statistical Service. 2010 Population and Housing census; summary report of final results. May 2017 projection. Nat. Data Arch (NADA). Available; http:// www.statsghana.gov.gh/docfiles/2010phc/Census2010_ Summary_report_of_final_results.pdf. Accessed on July 14, 2017.

9. Radner W. Near vision examination in Presbyopia patients: Do we need good homologated near vision charts? Eye Vis (Lond). 2016; 3: 29. doi: 10.1186/s40662016-0061-7

10. Jafarzadehpur E, Hashemi H, Abdollahinia T, et al., Design and Validation of Persian Near Reading Card: A pilot study. Irn J Ophthalmol. 2013;25:216-21.

11. Sailoganathan A, Siderov J, Osuobeni E. Relative Letter Legibility of Punjabi Optotypes. Ophthal Physiol Opt. 2002;22:573-82.

12. Sailoganathan, A., Siderov, J. and Osuobeni, E. Letter Legibility of Hindi and Sloan optotypes. Invest. Ophthalmol. Vis. Sci. 2003; 44, E-Abstract 2782.

13. Reeves BC, Wood JM, Hill AR. Reliability of High and Low Contrast Letter Charts. Ophthal Physiol Opt. 1993;13:17-26.

14. Lovie-Kitchin J. Validity and Reliability of Visual Acuity Measurements. Ophthal Physiol Opt. 1988;8:363-70. 15. Bailey IL, Lovie JE. New Design Principles for Visual Acuity Letter Charts. Am J Optom Physiol Opt. 1976;53:740-45.

16. Bailey IL, Bullimore MA, Raasch TW, Taylor HR. Clinical grading and the Effect of Scaling. Invest Opbthalmol Vis Sci. 1991;32:422-32.

17. Arditi A, Cagniello R. On the Statistical Reliability of Letter-Chart Visual Acuity Measurement. Invest Ophthalmol Vis Sci. 1993;34:120-29.

18. Vanden Bosch ME, Wall M. Visual Acuity Scored by the Letter-by-Letter or Probit Methods has Lower Retest Variability than the Line Assignment Method. Eye. 1997;11:411-17.

19. Elliott DB, Sheridan M. The Use of Accurate Visual Acuity Measurements in Clinical Anti-cataract Formulation Trials. Ophthal Physiol Opt. 1988;8:397-401.

20. Oduntan, A. O., and Al-Abdulmunem, M. A. Design of an Arabic near visual acuity chart. Ophthal Physiol. Opt. 1997; 17, 158-160.

21. Stifter E, König F, Lang T, et al. Reliability of a Standardized Reading Chart System: Variance Component Analysis, Test-Retest, and Inter-Chart Reliability. Graefes Arch Clin Exp Ophthalmol. 2004; 242:31-9.

22. Radner W, Obermayer W, Richter-Mueksch S, et al. The Validity and Reliability of Short German Sentences for Measuring Reading Speed. Graefes Arch Clin Exp Ophthalmol. 2002;240:461-7.

23. Legge GE, Ross JA, Isenberg LM, Lamay JM. Psychophysics of Reading.Clinical Predictors of Low Vision Reading Speed. Invest Ophthalmol Vis Sci. 1992;33:677-87. 